\title{
Simulating the Response of Terahertz Radiation to Basal Cell Carcinoma Using Double Debye model and FDTD Method
}

\author{
Mengxi Wang ${ }^{1,2}$, Guohui Yang ${ }^{1}$ and Qun $\mathrm{Wu}^{1 *}$ \\ ${ }^{1}$ Microwave Engineering Department, Harbin Institute of Technology, \\ Harbin, China 150001 \\ ${ }^{1}$ State Key laboratory of Millimeter Wave, Nanjing, China 210096 \\ ${ }^{2}$ The Fourth Affiliated Hospital, Harbin Medical University, Harbin, CHINA 150001 \\ qwu@hit.edu.cn,mengxi310@163.com
}

\begin{abstract}
Terahertz(THz) imaging provided a good contrast between skin cancer (basal cell carcinoma BCC) and healthy tissue in vitro and ex vivo owing to the high water content and strong absorption of cancer tissue at $\mathrm{THz}$ frequencies. Modeling the propagation of a $\mathrm{THz}$ pulse through BCC would contribute to revealing the diagnostic and potential therapeutic application value of $\mathrm{THz}$ radiation. In this letter, the healthy skin and BCC were modeled as Double Debye dispersive media and the model was incorporated into the FDTD method to simulate the propagation of a $\mathrm{THz}$ pulse. Furthermore, absorption properties were revealed by calculating average SAR value of the horizontal distribution and vertical distribution, which would help investigate the response below the skin surface.
\end{abstract}

Keywords: Terahertz; FDTD; Double Debye model; Basal cell carcinoma; Skin

\section{Introduction}

Human skin tissue has 3 layers: epidermis (100-200um), dermis and subcutaneous tissue. $\mathrm{BCC}$ as one of the most prevailing form of skin cancer, originates in the epidermal basal layer. The exposed surface of the cancer tissue could be easy to diagnose by clinical examination, however the invasion scope and depth located beneath the epidermis is difficult to judge (Figure 1) [1]. While the proper penetration capacity in skin (1 $\mathrm{mm}$ at $0.2 \mathrm{THz})$ [2] and a low photon energy enables $\mathrm{THz}$ to show great advantages in vivo detection and treatment of superficial tissue. Ruth M. et al., had revealed the ability of $\mathrm{THz}$ imaging to detect skin basal cell carcinoma (BCC) using Terahertz Pulse Imaging (TPI) system [3] based on the different hydration level of BCC and healthy skin. Work by Pickwell et al., has demonstrated that the terahertz response of normal human skin can be modeled in much the same way as water using a double Debye equation [4, 5], furthermore, they also extract Debye parameters (Table 1) from the refractive index and absorption coefficient. Recently, Heh using the Debye parameters and alternating-direction-implicit Finite-difference timedomain (FADI-FDTD) modeled the interaction of terahertz pulse with healthy skin and basal cell carcinoma (BCC)[6].Recent studies [7, 8] draw the attention of the potential therapeutic effect of $\mathrm{THz}$ in the field of cancer, which necessitates a better understanding of the interaction of terahertz pulse with healthy skin and BCC. 
Table 1. The Debye model parameters of healthy skin and BCC [5]

\begin{tabular}{cccccc}
\hline & $\varepsilon_{s}$ & $\varepsilon_{2}$ & $\varepsilon_{\infty}$ & $\tau_{1} / p s$ & $\tau_{2} / p s$ \\
\hline $\begin{array}{l}\text { health } \\
\text { y skin }\end{array}$ & 14.7 & 4.16 & 2.58 & 1.45 & 0.0611 \\
BCC & 17.6 & 4.23 & 2.65 & 1.55 & 0.0614 \\
\hline
\end{tabular}
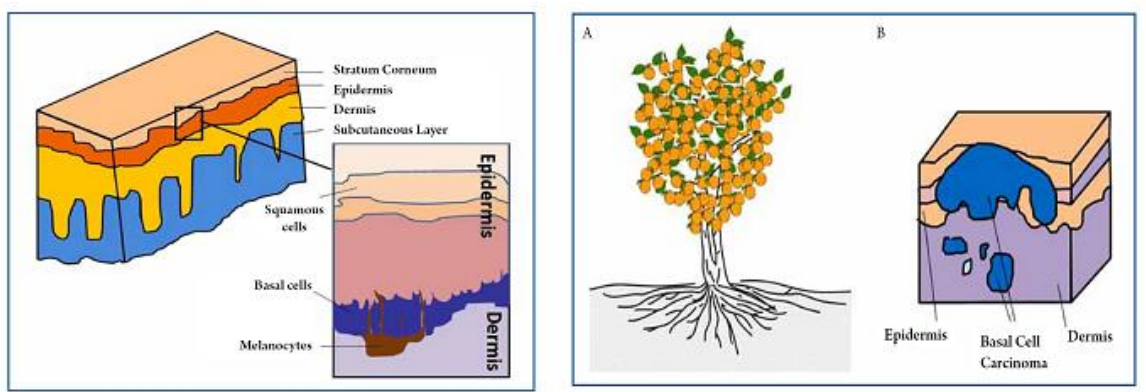

Figure 1. Schematic diagrams of the layers in human skin and the tree root analogy to basal cell carcinoma [1]

\section{FDTD Derivation of Dispersive Media}

The bio-organism, as a typical inhomogeneous magnet, is calculated to simulate by FDTD method $[9,10]$ when solving electromagnetic problem, which could divide the computational region into three-dimensional grids and assign electric parameters respectively.

The Maxwell equation for a dispersive media without a radiation source is expressed as:

$$
\left\{\begin{array}{l}
\nabla \times E=-\frac{\partial B}{\partial t}=-\mu \frac{\partial H}{\partial t} \\
\nabla \times H=\frac{\partial D}{\partial t}
\end{array}\right.
$$

Where $B=\mu H, \quad \mu$ is a constant.

Based on the Yee unit, FDTD derivation in the Cartesian coordinates of (1)could give the expression of each field component of $\mathrm{H}$ changing with time iteration step. The difference discrete processing of (2) in $t=(n+1 / 2) \Delta t$ is given by :

$$
(\nabla \times H)^{n+1 / 2}=\frac{D^{n+1}-D^{n}}{\Delta t}
$$

That is:

$$
D^{n+1}=D^{n}+\Delta t(\nabla \times H)^{n+1 / 2}
$$

$\tau_{2}$

In $\mathrm{THz}$ band, dispersive medium could be described by the Debye model theory with fast relaxation process model ( ) and slow relaxation process model ( ). The expression of the dielectric $\mathcal{E}_{s}$ oefficient varying with frequency is $\mathcal{E}_{\infty}$

$$
\hat{\varepsilon}(\omega)=\varepsilon_{\infty}+\frac{\varepsilon_{s}-\varepsilon_{2}}{1+j \omega \tau_{1}}+\frac{\varepsilon_{2}-\varepsilon_{\infty}}{1+j \omega \tau_{2}}
$$

Where is the static dielectric constant, is the high frequency limit, and is the intermediate frequency limit. 
According to the Maxwell equation of frequency constitutive relation, The relationship between $\mathrm{D}$ and $\mathrm{E}$ is :

$$
D(\omega)=\varepsilon_{0} \hat{\varepsilon}(\omega) \cdot E(\omega)
$$

Integrate (5) into (4):

$$
D(\omega)=\varepsilon_{0}\left[\varepsilon_{\infty}+\frac{\varepsilon_{s}-\varepsilon_{2}}{1+j \omega \tau_{1}}+\frac{\varepsilon_{2}-\varepsilon_{\infty}}{1+j \omega \tau_{2}}\right] E(\omega)
$$

That is :

$$
\begin{aligned}
&\left(1-\omega^{2} \tau_{1} \tau_{2}\right) \frac{D^{n+1}+D^{n}}{2}+\left(\tau_{1}+\tau_{2}\right) \frac{D^{n+1}-D^{n}}{\Delta t} \\
&= \tau_{2} \varepsilon_{\infty} \frac{E^{n+1}-E^{n}}{\Delta t}-\tau_{1} \tau_{2} \omega^{2} \varepsilon_{\infty} \frac{E^{n+1}+E^{n}}{2}+\varepsilon_{\infty} \frac{E^{n+1}+E^{n}}{2}+ \\
& \tau_{2} \varepsilon_{s} \frac{E^{n+1}-E^{n}}{\Delta t}-\tau_{2} \varepsilon_{2} \frac{E^{n+1}-E^{n}}{\Delta t}+\tau_{1} \varepsilon_{2} \frac{E^{n+1}-E^{n}}{\Delta t}
\end{aligned}
$$

The transformation relation between frequency domain and the time domain operator $j \omega=\partial / \partial t$ leads to :

$$
\begin{aligned}
& \left(1-\omega^{2} \tau_{1} \tau_{2}\right) D(\omega)+\left(\tau_{1}+\tau_{2}\right) \frac{\partial D}{\partial t} \\
& =\tau_{2} \varepsilon_{\infty} \frac{\partial E}{\partial t}-\tau_{1} \tau_{2} \omega^{2} \varepsilon_{\infty} E(\omega)+\varepsilon_{\infty} E(\omega)+\tau_{2} \varepsilon_{s} \frac{\partial E}{\partial t}-\tau_{2} \varepsilon_{2} \frac{\partial E}{\partial t}+\tau_{1} \varepsilon_{2} \frac{\partial E}{\partial t}
\end{aligned}
$$

(1-8) is the time domain differential equations of $\mathrm{D}$ and E,corresponding with the Debye formula (1-4) . (1-8) dispersed at time $t=(n+1 / 2) \Delta t$ becomes:

$$
\begin{aligned}
& \left(1-\omega^{2} \tau_{1} \tau_{2}\right) D(\omega)+j \omega\left(\tau_{1}+\tau_{2}\right) D(\omega) \\
& =j \omega \tau_{2} \varepsilon_{\infty} E(\omega)-\tau_{1} \tau_{2} \omega^{2} \varepsilon_{\infty} E(\omega)+\varepsilon_{\infty} E(\omega)+ \\
& j \omega \tau_{2} \varepsilon_{s} E(\omega)-j \omega \tau_{2} \varepsilon_{2} E(\omega)+j \omega \tau_{1} \varepsilon_{2} E(\omega)
\end{aligned}
$$

The above formulas could be transformed into :

$$
\begin{aligned}
& \left(\frac{\tau_{1}+\tau_{2}}{\Delta t}+\frac{1-\omega^{2} \tau_{1} \tau_{2}}{2}\right) D^{n+1}-\left(\frac{\tau_{1}+\tau_{2}}{\Delta t}-\frac{1-\omega^{2} \tau_{1} \tau_{2}}{2}\right) D^{n} \\
= & \left(\frac{\tau_{2} \varepsilon_{\infty}+\varepsilon_{\infty} \tau_{2}-\tau_{2} \varepsilon_{2}+\tau_{1} \varepsilon_{2}}{\Delta t}+\frac{\varepsilon_{\infty}-\tau_{1} \tau_{2} \omega^{2} \varepsilon_{\infty}}{2}\right) E^{n+1}- \\
& \left(\frac{\tau_{2} \varepsilon_{\infty}+\varepsilon_{\infty} \tau_{2}-\tau_{2} \varepsilon_{2}+\tau_{1} \varepsilon_{2}}{\Delta t}-\frac{\varepsilon_{\infty}-\tau_{1} \tau_{2} \omega^{2} \varepsilon_{\infty}}{2}\right) E^{n}
\end{aligned}
$$

$\mathrm{D}$ to $\mathrm{E}$ conversion of the time domain becomes:

Where:

$$
E^{n+1}=C U \cdot E^{n}+C V \cdot D^{n+1}-C W \cdot D^{n}
$$

$$
\left\{\begin{array}{l}
C U=\frac{\frac{\tau_{2} \varepsilon_{\infty}+\varepsilon_{\infty} \tau_{2}-\tau_{2} \varepsilon_{2}+\tau_{1} \varepsilon_{2}}{\Delta t}-\frac{\varepsilon_{\infty}-\tau_{1} \tau_{2} \omega^{2} \varepsilon_{\infty}}{2}}{\frac{\tau_{2} \varepsilon_{\infty}+\varepsilon_{\infty} \tau_{2}-\tau_{2} \varepsilon_{2}+\tau_{1} \varepsilon_{2}}{\Delta t}+\frac{\varepsilon_{\infty}-\tau_{1} \tau_{2} \omega^{2} \varepsilon_{\infty}}{2}} \\
C V=\frac{\frac{\tau_{1}+\tau_{2}}{\Delta t}+\frac{1-\omega^{2} \tau_{1} \tau_{2}}{2}}{\frac{\tau_{2} \varepsilon_{\infty}+\varepsilon_{\infty} \tau_{2}-\tau_{2} \varepsilon_{2}+\tau_{1} \varepsilon_{2}}{\Delta t}+\frac{\varepsilon_{\infty}-\tau_{1} \tau_{2} \omega^{2} \varepsilon_{\infty}}{2}} \\
C W=\frac{\frac{\tau_{1}+\tau_{2}}{\Delta t}-\frac{1-\omega^{2} \tau_{1} \tau_{2}}{2}}{\frac{\tau_{2} \varepsilon_{\infty}+\varepsilon_{\infty} \tau_{2}-\tau_{2} \varepsilon_{2}+\tau_{1} \varepsilon_{2}}{\Delta t}+\frac{\varepsilon_{\infty}-\tau_{1} \tau_{2} \omega^{2} \varepsilon_{\infty}}{2}}
\end{array}\right.
$$

When (1-7) is dispersed in space, based on Yee unit, each component of $E$ in the time domain could be obtained step by step. So, solving processes of FDTD changing with time in 
the dispersive medium are as follows:

(1) Calculate each components of $H^{n+1 / 2}$ by E in FDTD formulas

(2) Calculate each component of $D^{n+1}$ by $\mathrm{H}$ according to (1-3)

(3) Calculate each component of $E^{n+1}$ by $\mathrm{D}$ according to (1-11)

(4) go back to step (1).

\section{Interaction of Terahertz Pulse with Healthy Skin and BCC}

\subsection{Skin BCC model}

The model in this paper consisted of two parts: 300um thickness of health skin and BCC embedded in healthy tissue of the same thickness, assuming that BCC had affected the whole layer of epidermis, and had been extended to the subepidermal dermis. The receiving surface was chosen to be $420 \times 420 \mathrm{um}$, the cross section of BCC is round (radius 150um)[6] (Figure 2).
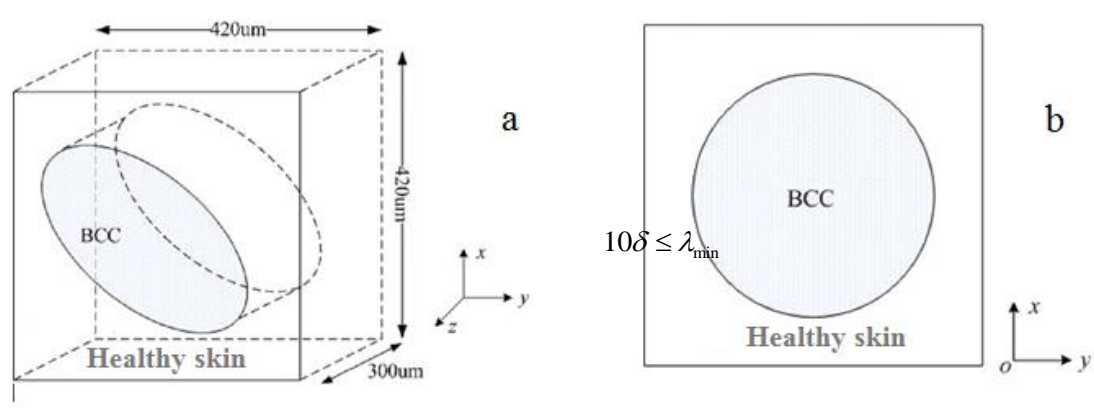

Figure 2 skin model with embedded BCC tissue.

a) Schematic diagram of the whole structure, b)Top view

\subsection{Parameter settings}

2.2.1 Space Mesh: The spatial grids were composed of basic unit of grid ,hexahedral shape, hence $\Delta x=\Delta y=\Delta z=\delta$. In order to reduce the numerical dispersion , length $\delta$ of the basic unit should meet the following conditions: wavelength of the calculation space, and $\Delta x=\Delta y=\Delta z=\delta=6 \mathrm{~mm}$.

2.2.2 Time Step: When $\Delta x=\Delta y=\Delta z=\delta$, in order to ensure numerical stability, the time step $\Delta t$ should satisfy the condition: $c \Delta t \leq \frac{\delta}{\sqrt{3}} \quad$. Limit of time step is commonly defined as: $c \Delta t \leq \frac{\delta}{2}$.
Therefore, $t=0.01 \mathrm{ps}$.

2.2.3 Space Size: The axial length of the space was: $L_{x}=N_{x} \square \boldsymbol{x}, \boldsymbol{L}_{y}=N_{y} \square y, L_{z}=N_{z} \square z$. The total number of cells along the three coordinate axes were $\mathrm{Nx}, \mathrm{Ny}, \mathrm{Nz}$ respectively, usually, about 6-10 grid basic units was set between the connecting boundary and absorbing boundary, and 10 grid units were selected in this study.

2.2.4 Absorbing Boundary: The two order Mur absorbing boundary was used this subject. 
2.2.5 Source Settings: The improved Gauss pulse with frequency of $1.0 \mathrm{THz}$ and pulse width being 32 times of $\Delta \mathrm{t}$ was used in this project, and time domain and frequency domain waveform were as shown in Figure 3.

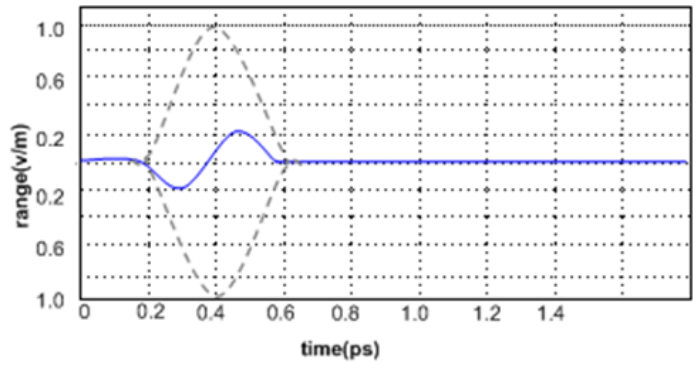

a) Time domain waveform

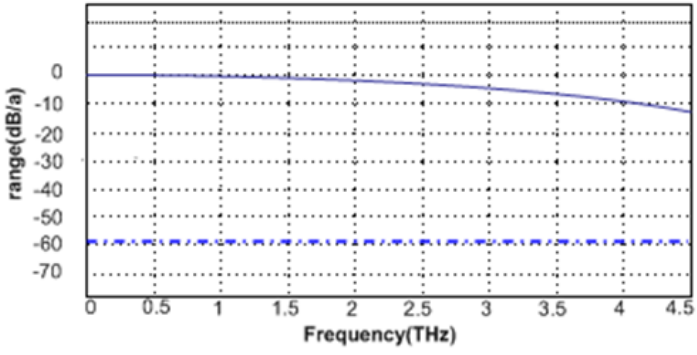

b) Frequency domain waveform

Figure 3. Waveform of improved Gauss pulse

\subsection{Time Step Number Nt}

In order to estimate $N_{t}$, the steady state of distribution in the calculated area was required. In general, we believed that after the electromagnetic waves passing through space diagonals 3 to 5 times, the distribution of electromagnetic field could be considered as stable. The total number of units of the calculation space was $\mathrm{N}$, so the units on a diagonal was $\sqrt{3} N^{1 / 3}$. According to the Courant stability condition, we $\operatorname{set}_{\Delta t}=\delta /(2 c)$, so the number of time steps was $2 \sqrt{3} N^{1 / 3}$ when waves across a diagonal, thus the total number of step was $2 \sqrt{3} \times(6 \square 10) N^{1 / 3}$. As a result, the final time step number was set $N_{t}=1000$.

\section{The FDTD Simulation Results}

The model was simulated with the parameters above, the average SAR of xoy plane when $\mathrm{Nz}=10$ was observed in Figure 4. The red region was representative of the maximum SAR value of $\mathrm{BCC}$, proving that $\mathrm{THz}$ absorption of $\mathrm{BCC}$ was higher than other part in consequence of the higher water content of BCC [1]. As was calculated, SAR maximum value was $2.89812 \mathrm{~W} / \mathrm{kg}$, and the average SAR was $0.299592 \mathrm{~W} / \mathrm{kg}$. Figure 5 showed the average SAR distribution of yoz plane centered at $\mathrm{Nx}=70$ vertical section, where the absorption of SAR decreased gradually from up to down, The red area, BCC tissue, indicated stronger absorption, and, from another perspective, faster attenuation of $\mathrm{THz}$ wave than healthy tissue .

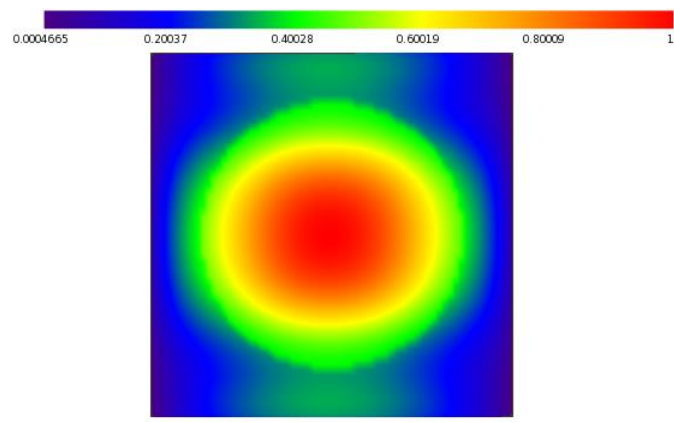

Figure 4. $\mathrm{N}_{\mathrm{z}}=10$ average SAR distribution of yoz plane $(\mathrm{W} / \mathrm{kg})$

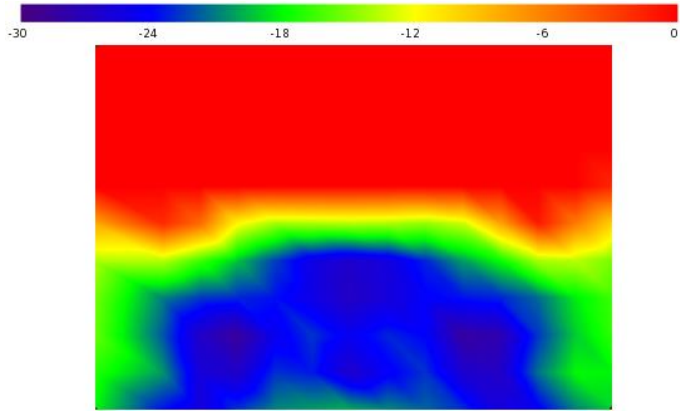

Figure 5. $\mathrm{N}_{\mathrm{x}}=70$ average SAR distribution of yoz plane $(\mathrm{dB})$ 


\section{Conclusion}

This paper has presented the application of FDTD method in modeling the interaction of terahertz pulse with healthy skin and BCC. The healthy skin and BCC have been modeled as Debye dispersive media and the model is incorporated into the FDTD method, which help provide a deeper understanding of the way in which terahertz radiation propagates through BCC. Further research on complex model of tissues and cells under conditions of different power lever, irradiation time, frequency, sphere of action,etc,may be able to lead us to find the specific data to destruct BCC cells .

\section{References}

[1] C. Yu, S. Fan, Y. Sun and E. Pickwell-MacPherson, Quantitative Imaging in Medicine and Surgery, vol. 2, no. 1, (2012).

[2] V. P.Wallace, A. J. Fitzgerald, E. Pickwell, R. J. Pye, P. F. Taday, N. Flanagan and T. Ha, Appled Spectroscpy, vol. 60, no. 10, (2006).

[3] R. M. Woodward, V. P. Wallace, R. J. Pye, B. E. Cole, D. D. Arnone, E. H. Linfield and M. Pepper, The Journal Of Investigative Dermatology, vol. 120, no. 1, (2003).

[4] E. Pickwell, B. E. Cole, A. J. Fitzgerald, V. P. Wallace and M. Pepper, Appl. Phys. Lett., vol. 84, no. 12, (2004).

[5] E. Pickwell, A. J. Fitzgerald, B. E. Cole, P. F. Taday, R. J. Pye, T. Ha, M. Pepper and V. P. Wallace, J. Biomed. Opt., vol. 10, no. 6, (2005).

[6] D. Y. Heh and E. L. Tan, Progress In Electromagnetics Research B, vol. 37, (2012).

[7] G. J. Wilmink, B. D. Rivest, C. C. Roth, B. L. Ibey, J. A. Payne, L. X. Cundin, J. E. Grundt, X. Peralta, D. G. Mixon and W. P. Roach, Lasers Surg. Med., vol. 43, (2011).

[8] P. Maniadis, B. S. Alexandrov, A. R. Bishop and K. O. Rasmussen, Rev. E Stat. Nonlin. Soft Matter Phys., vol. 83 , no. $1,(\mathbf{2 0 1 1})$.

[9] K. S. Yee, "Numerical solution of initial boundary value problems involving Maxwell's equation in isotropic media", Antennas and Propagation, IEEE Transactions on In Antennas and Propagation, vol. 14, no. 4, (1966), pp. 302-307.

[10] A. Taflove and S. C. Hagness, "Computational Electrodynamics:The Finite-Difference Time-Domain Method”, Artech House, Boston, MA, (2005). 